\title{
Plasma-Wall Interaction Study towards the Steady State Operation
}

\author{
Mizuki SAKAMOTO, Yuta HIGASHIZONO, Hideki ZUSHI, Kazuo NAKAMURA, \\ Kazuaki HANADA, Hiroshi IDEI, Makoto HASEGAWA, Younosuke NAKASHIMA ${ }^{1)}$, \\ Masayuki TOKITANI ${ }^{2)}$, Mitsutaka MIYAMOTO ${ }^{3)}$, Kazutoshi TOKUNAGA, Shoji KAWASAKI, \\ Hisatoshi NAKASHIMA, Tadashi FUJIWARA, Aki HIGASHIJIMA, Naoaki YOSHIDA \\ and Kohnosuke SATO \\ Research Institute for Applied Mechanics, Kyushu University, 6-1 Kasuga-koen, Kasuga, Fukuoka 816-8580, Japan \\ ${ }^{1)}$ Plasma Research Center, Tsukuba University, Ibaraki 305-8577, Japan \\ ${ }^{2)}$ National Institute for Fusion Science, 322-6 Oroshi-cho, Toki, Gifu 509-5292, Japan \\ ${ }^{3)}$ Department of Material Science, Shimane University, Matsue, Shimane 690-8504, Japan
}

(Received 21 December 2009 / Accepted 29 March 2010)

\begin{abstract}
Various phenomena of plasma-wall interactions during long duration discharges in TRIAM-1M are investigated from macroscopic and microscopic viewpoints. It is found that the density dependence of the hydrogen neutral flux decay length is not very sensitive to the density (i.e. $\bar{n}_{\mathrm{e}}^{-0.2}$ ). Neutral transport through the scrape-off layer is important for structural formation of hydrogen recycling. The hydrogen retention in the co-deposited layer, which was obtained using a material probe during long duration discharges, is consistent with the global wall pumping rate estimated from particle balance analysis. The wall temperature and co-deposition play important roles on hydrogen re-emission and hydrogen absorption, respectively. Oxygen impurities should affect the erosion and deposition properties of the plasma-facing wall as well as the hydrogen retention property of the co-deposited layer. The co-deposition of hydrogen with molybdenum and the oxygen impurities both play important roles in the hydrogen recycling.
\end{abstract}

(C) 2010 The Japan Society of Plasma Science and Nuclear Fusion Research

Keywords: plasma-wall interaction, steady state operation, tokamak, hydrogen recycling, co-deposition

DOI: $10.1585 /$ pfr.5.S2009

\section{Inroduction}

Plasma-wall interaction (PWI) issues are strongly related to plasma performance and stable operation, and are especially critical for a steady state operation (SSO) of fusion plasma [1-3]. PWI phenomena contain processes over a wide range of time and length scales. The time scale characteristics of PWI extend from 10 ps (radiation damage) to $\leq \mathrm{ms}$ (hydrogen diffusion in material), order of 10 's seconds (recycling variation) and order of $10^{2}-10^{3}$ seconds (wall saturation) up to order of $10^{6}$ (life time of plasmafacing components). On the other hand, the characteristics of the PWI length scale range from the nm order (microstructure) to tens of $\mathrm{m}$ order (global particle balance) with $\mu \mathrm{m}$ (dust) and cm (hot spot) orders in between. Therefore, PWI phenomena must be comprehensively investigated from multiscale viewpoints because the scale length phenomena are interrelated.

In TRIAM-1M, which has SSO capability, plasmawall interaction experiments have been carried out extensively from microscopic and macroscopic viewpoints [4-17]. In this paper, various phenomena of PWI are investigated focusing on their length scales, which are classified according to the length from the order of a diameter of the torus to that of the microstructure of the deposits on

author's e-mail: sakamoto@triam.kyushu-u.ac.jp the wall.

\section{Experimental Setup}

TRIAM-1M is a superconducting tokamak. Sixteen superconducting toroidal field coils composed of $\mathrm{Nb}_{3} \mathrm{Sn}$ were installed. The toroidal magnetic field was up to $8 \mathrm{~T}$ and in the steady state. The poloidal field coils of $\mathrm{Cu}$ were mounted on a vacuum vessel with a $\mathrm{D}$-shaped cross-section, a horizontal length of $0.26 \mathrm{~m}$, and the vertical length of $0.38 \mathrm{~m}$. The major radius of the center of the vacuum vessel was $0.84 \mathrm{~m}$. The entire machine was installed inside a bell-shaped vacuum vessel (i.e. a belljar) for thermal insulation. Extension ports connected the plasma vacuum vessel and the bell-jar.

The plasma-facing components were made of high $Z$ materials: three fixed poloidal limiters (PLs) and divertor plates were made of molybdenum, and the vacuum vessel (i.e. main chamber) was stainless steel as shown in Fig. 1. A movable limiter (ML) with a front edge made of molybdenum was installed in the same section as the fixed poloidal limiter and the pumping port. The ML was thermally insulated from the main chamber, and had a good cooling capability. A low $Z$ material coating was not applied. Wall conditioning was performed just prior to the 


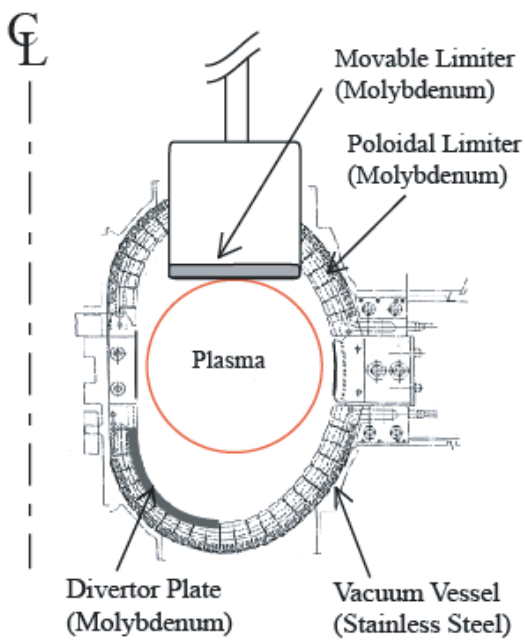

Fig. 1 Cross-sectional view of the main chamber at the section of the movable limiter and the fixed poloidal limiter.

experimental campaign. The extension ports were initially heated to $100^{\circ} \mathrm{C}$ for $\sim 2$ days, and then electron cyclotron resonance discharge cleaning for $\sim 2$ days was carried out to clean the plasma-facing components $[18,19]$. To avoid a thermal load to the cryogenic system, the plasma vacuum vessel was not heated. The main chamber was evacuated using a turbo-molecular-pump with an effective pump speed of $5501 \mathrm{~s}^{-1}$.

The plasma current was initiated by ohmic heating ( $\tau \sim 0.2 \mathrm{~s})$, and then sustained by a lower hybrid current drive (LHCD) with a frequency of $2.45 \mathrm{GHz}$ or $8.2 \mathrm{GHz}$. The hydrogen gas fueling was controlled by feedback to match the $\mathrm{H}_{\alpha}$ intensity at the central chord with a reference signal. It should be noted that all the results reported herein were obtained from hydrogen discharges with a limiter configuration.

\section{Experimental Results and Discus- sion}

\subsection{Hydrogen recycling}

In TRIAM-1M, the recycling coefficient $(R)$ was evaluated by using a particle balance equation inside the plasma [6]. The value of $R$ increased with the discharge duration and approached to unity. In the first one minute, there existed two time constants of the increase in $R$ : one was a few $\mathrm{s}$ and the other was $\sim 30 \mathrm{~s}$. These time constants may relate to implantation of hydrogen into the wall and diffusion of hydrogen in the wall, respectively. It was also found the third time constant for the variation of hydrogen recycling that was $\sim 30$ min or longer (i.e. nearly infinity). The third time constant is explained in the section 3.3.

\subsection{Neutral behavior}

Figure 2 (a) shows a toroidal profile of the $\mathrm{H}_{\alpha}$ intensity $\left(I_{\mathrm{H \alpha}}\right)$, which was measured at $120 \mathrm{~s}$ after plasma initiation. The plasma was sustained by $8.2 \mathrm{GHz}$ LHCD with
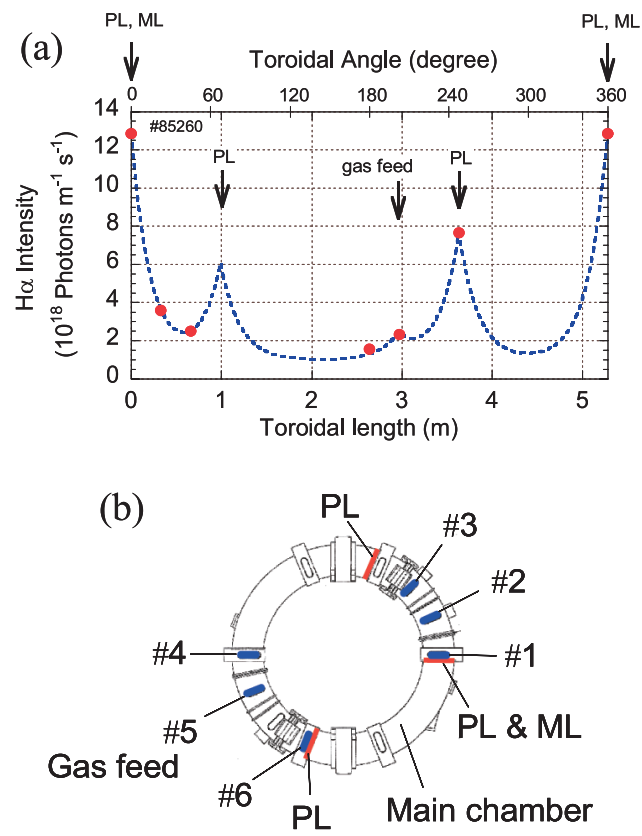

Fig. 2 (a) Toroidal profile of $\mathrm{H}_{\alpha}$ line intensity and (b) top view of the main chamber. Three sets of the poloidal limiters (PL) are installed. Movable limiter (ML) is installed in section \#1. Hydrogen gas is injected under the torus in section \#5. $\mathrm{H}_{\alpha}$ intensity measured in sections \#1-\#6.

a power of $40 \mathrm{~kW}$. The line averaged electron density $\left(\bar{n}_{\mathrm{e}}\right)$ was $\sim 0.6 \times 10^{19} \mathrm{~m}^{-3}$. It was found that $I_{\mathrm{H} \alpha}$ at the PL and the ML became rather high. As shown by the broken line in Fig. 2 (a), the profile is well reproduced according to the following fitting equation:

$$
I_{\mathrm{H} \alpha}(x)=P_{\mathrm{mc}}+\sum_{i=1}^{4} P_{i} \exp \left[\frac{-\left|x-x_{i}\right|}{\lambda}\right],
$$

where $x$ is the toroidal length. $P_{\mathrm{mc}}$ is the contribution of main chamber recycling, and is assumed to be homogeneous in the toroidal direction. The second term in the right-hand side represents the contribution of the limiters and gas feed. $\lambda$ is the decay length of $I_{\mathrm{H} \alpha}$ from the source of hydrogen neutrals, and denotes the mean-free path of hydrogen neutral flux in the torus. $P_{\mathrm{mc}}, P_{\mathrm{i}}$ and $\lambda$ are determined as the profile of eq. (1) agrees with the experimental data that are shown by red closed circles in Fig. 2 (a). In the case of Fig. 2 (a), $\lambda$ is $0.21 \mathrm{~m}$. The contribution ratio of the main chamber, the ML\&PL section, other PLs, and gas feed to $I_{\mathrm{H} \alpha}$ were $34 \%, 32 \%, 31 \%$, and $3 \%$, respectively. It was found that hydrogen recycling in the main chamber and the limiters dominated, and the contribution of the gas feed was negligible because the recycling ratio became higher as the discharge duration increased [6].

Figure 3 (a) shows the dependence of $\lambda$ on $\bar{n}_{\mathrm{e}}$. The value of $\lambda$ gradually decreased as $\bar{n}_{\mathrm{e}}$ increased (i.e. $\lambda \propto$ $\bar{n}_{\mathrm{e}}^{-0.2}$ ), although a simple model of the mean-free path suggests that $\lambda$ varies inversely with the density. The density dependence of $\lambda$ can be well reproduced by a DE- 
(a)

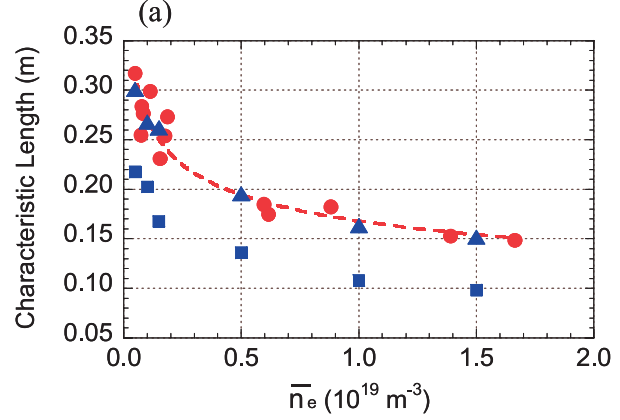

(b)

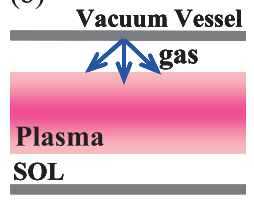

model-A (c)

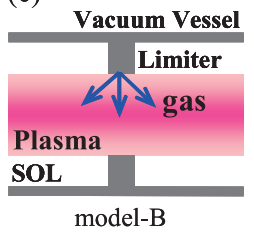

Fig. 3 (a) Density dependence of the decay length of the $\mathrm{H}_{\alpha}$ intensity estimated using the equation (1). Closed triangles and squares are decay lengths estimated by DEGAS simulation using cylindrical models (b) and (c), respectively.

GAS simulation [20] using a cylindrical model, model-A in Fig. 3 (b), where the neutral source is located on the wall. Typical profiles of the plasma density and temperature in $2.45 \mathrm{GHz}$ LHCD were used for the DEGAS simulation. Temperature of the scrape off layer (SOL) is assumed to be $30 \mathrm{eV}$ in each density in Fig. 3 (a). One candidate of a reason of the weak dependence of $\lambda$ on $\bar{n}_{\mathrm{e}}$ may be that the mean-free path of the neutral hydrogen becomes substantially extended due to increase in charge exchange neutrals with higher energy than Frank-Condon energy as the density increases.

In contrast, another model, model-B in Fig. 3(c), where the ring limiter is set around the plasma did not reproduce the density dependence of $\lambda$ as indicated in Fig. 3 (a). In model-B, the ring limiter blocked neutral transport through SOL. In the case of TRIAM-1M, although the recycling neutral flux was mainly due to the limiters as shown in Fig. 2 (a), a sufficient space existed between the plasma and the limiter (Fig. 1). Hence, the neutral particles can be transported through the space during recycling, indicating that the transport of neutrals through SOL and the plasma periphery plays an important role in structure formation of hydrogen recycling.

\subsection{Global particle balance}

Hydrogen recycling in long duration discharges has been studied using global particle balance analysis $[6,9$, 21-24]. The global particle balance in the main chamber can be written as:

$$
\frac{\mathrm{d} N_{\mathrm{H}}^{0}}{\mathrm{~d} t}+\frac{\mathrm{d} N_{\mathrm{H}}^{p}}{\mathrm{~d} t}=\Gamma_{\text {fuel }}-\Gamma_{\text {pump }}-\Gamma_{\text {wall }},
$$

where $N_{\mathrm{H}}^{0}$ is the total number of neutral hydrogen atoms
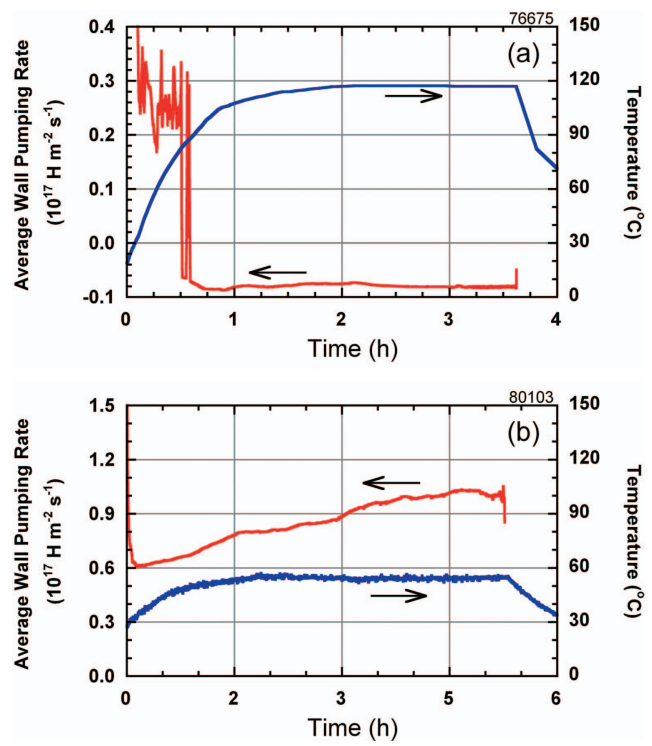

Fig. 4 Time evolution of the average wall pumping rate and wall temperature in ultra-long duration discharges (a) with and (b) without a movable limiter. Plasma density is deduced to be $\sim 1 \times 10^{18} \mathrm{~m}^{-3}$ for both discharges. RF power is $\sim 7$ $\mathrm{kW}$ and $\sim 6 \mathrm{~kW}$ for the discharges of (a) and (b), respectively.

in the chamber. $N_{\mathrm{H}}^{p}$ is the total number of hydrogen ions in the plasma. $\Gamma_{\text {fuel }}$ is the fueling rate, $\Gamma_{\text {pump }}$ is the pumping rate by the external pump-unit, and $\Gamma_{\text {wall }}$ is the net wall pumping rate. Under steady state conditions, $\Gamma_{\text {wall }}$ can simply be obtained by the balance between $\Gamma_{\text {fuel }}$ and $\Gamma_{\text {pump }}$ because the left-hand side becomes zero. Figure 4 shows the time evolution of the average wall pumping rate and the wall temperature for two types of long duration discharges. The major difference between discharges was the wall temperature. In the discharge of Fig. 4 (a), the ML was not employed, and the wall temperature increased partly up to $120^{\circ} \mathrm{C}$ due to the heat load from the plasma. On the other hand, in the discharge of Fig. 4(b), the increase in the wall temperature was suppressed to less than $60^{\circ} \mathrm{C}$ using the ML. Calorimetry measurements revealed that ML deals with $34 \%$ of the entire heat load. The wall temperature was measured at a back side of the main chamber using thermocouples. It should be noted that the temperature distribution of the main chamber ranged from room temperature to the maximum value shown in Fig. 4. This inhomogeneous temperature distribution is attributed to geometry of the cooling water pipes, which are welded on the back side of the main chamber, as well as heat load distribution from the plasma. The maximum temperature was measured at a bellows part of the main chamber that was not directly cooled.

As shown in Fig. 4 (a), the average wall pumping rate was $2.4 \times 10^{16} \mathrm{H} \mathrm{m}^{-2} \mathrm{~s}^{-1}$ in the first 30 minutes, but then changed to a negative value $\left(-8 \times 10^{15} \mathrm{H} \mathrm{m}^{-2} \mathrm{~s}^{-1}\right)$, implying that the wall serves as both a particle sink and parti- 
cle source during discharge. The change of the wall's role from a particle sink to source is called "wall saturation." After $t \sim 17 \mathrm{~min}$ a process of fueling and no fueling was repeated to keep the $\mathrm{H}_{\alpha}$ intensity constant by feedback. This seems to mean that the wall approached the global saturation although the average wall pumping rate was almost constant before the global wall saturation occurred. It is noted that the wall saturation does not always mean the whole wall cannot absorb the hydrogen. The wall pumping rate means an integral of a balance between a hydrogen absorption rate $\left(\Gamma_{\mathrm{ab}}\right)$ and a re-emission rate $\left(\Gamma_{\mathrm{re}}\right)$ and is written as

$$
\Gamma_{\text {wall }}=\int_{S} \Gamma_{\mathrm{ab}}-\Gamma_{\mathrm{re}} \mathrm{d} a,
$$

where $S$ is a whole wall surface. The wall can locally absorb hydrogen even though the wall saturation occurs globally. In the case of Fig. 4(a), the global wall saturation seems to be attributed to the increase in the re-emission of hydrogen from the wall due to the increase in the wall temperature. Due to the increase in the re-emission, the $\mathrm{H}_{\alpha}$ intensity slowly increased with the time constant of $\sim 1000 \mathrm{~s}$ with no external fueling after $t \sim 30 \mathrm{~min}$.

In contrast, as shown in Fig. 4 (b), the wall continued to absorb hydrogen until the end of the discharge ( $t \sim 5 \mathrm{~h} 16 \mathrm{~min}$ ) when the ML was inserted into the plasma edge. In this discharge, the average wall pumping rate was $\sim 8.6 \times 10^{16} \mathrm{H} \mathrm{m}^{-2} \mathrm{~s}^{-1}$, which is 3.6 times higher than that in Fig. 4 (a). This means that the hydrogen re-emission from the wall is suppressed due to a smaller temperature increase observed in Fig. 4(b). As discussed in the subsequent section, this continuous wall pumping should be attributed to the co-deposition of hydrogen with Mo sputtered from the divertor plates.

\subsection{Impurity behavior}

As mentioned in the previous section, the codeposition of hydrogen with molybdenum may play an important role on the continuous wall pumping in a long duration discharge. In order to study the properties of erosion and deposition of plasma-facing materials, a real time, in situ measurement system for erosion and deposition has been developed in TRIAM-1M [17]. This system is based on light interference on a thin semi-transparent layer of redeposited material on the substrate [25]. A $4 \mathrm{~mm}$ thick sapphire window was used as a substrate, which was also used for Thomson scattering measurement. The window was about $75 \mathrm{~mm}$ from the last closed flux surface of the plasma that was at the center of the poloidal limiter. A fiber optic bundle, which was composed of 450 optic fibers with a $100 \mu \mathrm{m}$ diameter, was attached to the air side of the window. The optic fibers were mixed statistically. The fiber optic bundle was pushed on the window surface by spring action to avoid a gap between the bundle and window due to vibrations during the plasma production. Half of the optic fibers were used to illuminate the substrate with laser

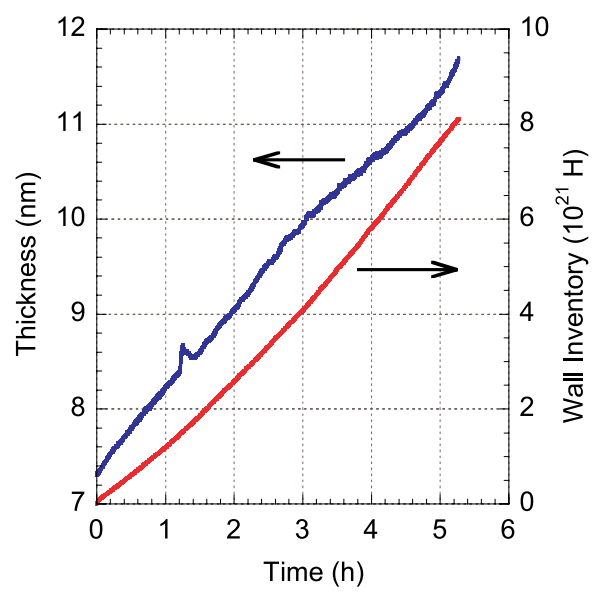

Fig. 5 Time evolution of deposition layer growth for Mo atoms sputtered on the divertor plate and the wall inventory.

light $(\lambda \sim 635 \mathrm{~nm})$, while the other half guided the reflected light back to a photodiode. In order to avoid plasma light, an interference filter was mounted in front of the photodiode. The intensity of the laser light was monitored by the other photodiode.

Figure 5 shows the time evolution of growth of the deposited layer and the wall inventory in the 5-h discharge shown in Fig. 4(b). The wall inventory was obtained by temporal integration of the wall pumping rate shown in Fig. 4 (b). The average growth rate of the deposited layer was $\sim 2.3 \times 10^{-4} \mathrm{~nm} \mathrm{~s}^{-1}$, i.e. $\sim 1.5 \times 10^{16} \mathrm{Mo} \mathrm{m}^{-2} \mathrm{~s}^{-1}$. The wall inventory reached $\sim 8 \times 10^{21} \mathrm{H}$. Both the wall inventory and growth of the deposition layer indicated a similar tendency. Although the spatial deposition profile on the plasma-facing component is necessary for quantitative analysis, the monotonic increase of the deposited layer suggests that continuous wall pumping is attributed to the co-deposition of hydrogen with sputtered Mo atoms.

Figure 6 (a) shows the deposition pattern on a sapphire window after the last experimental campaign in 2005. The experimental period was 1.5 month. The cross in Fig. 6 (a) indicates the measurement position of erosion and deposition. The thickest part of the deposited layer $(>20 \mathrm{~nm})$ was located at the lower left, and the deposition pattern was asymmetric relative to the mid-plane of the vacuum vessel. The likely molybdenum source for the deposition on the sapphire window was the divertor plate, which was located at the bottom of the vacuum vessel. The divertor plate was sputtered by charge exchange neutral hydrogen, and some of the sputtered molybdenum atoms can reach the sapphire window. A previous experiment has reported that all the deposits formed on a specimen placed in a vacuum vessel for the duration of an experimental campaign were molybdenum, and the major elemental components (i.e. iron, chromium, and nickel) of the vacuum vessel were not detected [4]. Figure 6(b) shows the angular distribution of the sputtered molybdenum atoms from the divertor plate in the positive $x$ direction. The length from the origin 


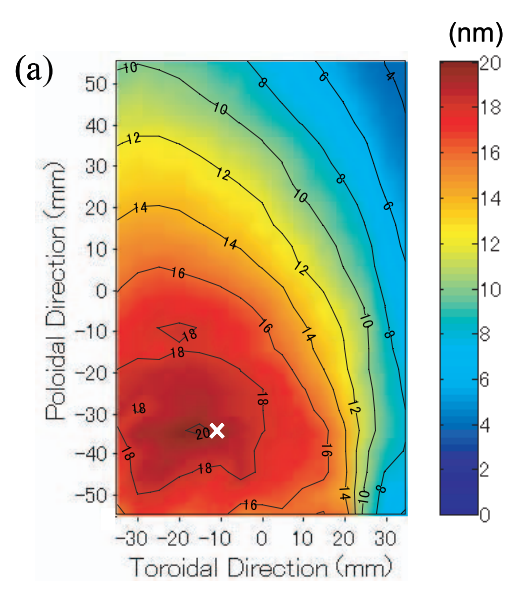

(b)

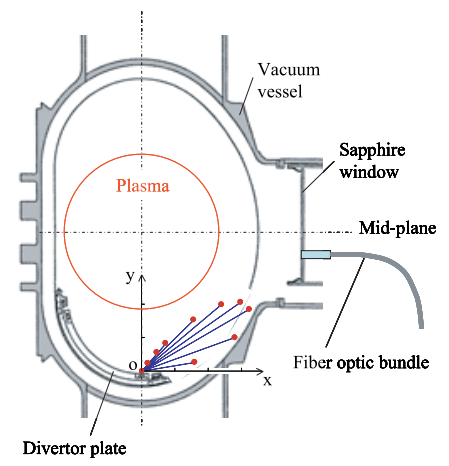

Fig. 6 (a) Mo deposition pattern on the sapphire window after the last experimental campaign in 2005. (b) Diagram of the angular distribution of sputtered Mo atoms from the divertor plate superimposed on the cross-section of the vacuum vessel.

corresponds to the number of molybdenum atoms capable of reaching the wall through the SOL plasma. Because the molybdenum atoms, which reach the upper part of the window, must pass through a higher density and higher temperature region and its pass length becomes longer, fewer molybdenum atoms can reach the upper part of the window. This may be the reason for the up-down asymmetry of the deposition pattern. As for the toroidal direction, the deposition pattern was weighted toward the ion drift side, suggesting anisotropic sputtering in the toroidal direction.

The net erosion and deposition rate on the window depended on the discharge conditions, and ranged between $-2 \times 10^{-3} \mathrm{~nm} \mathrm{~s}^{-1}$ to $2 \times 10^{-3} \mathrm{~nm} \mathrm{~s}^{-1}$ for long duration discharges $\left(0.1 \times 10^{19} \mathrm{~m}^{-3} \leq \bar{n}_{\mathrm{e}} \leq 1 \times 10^{19} \mathrm{~m}^{-3}\right)$. The positive and negative signs indicate deposition and erosion, respectively. Figure 7 shows a typical result in a high power and high density discharge in $8.2 \mathrm{GHz}$ LHCD. In the latter phase of the discharge, the plasma density increased due to enhanced PWI, although the gas feed was terminated. The net RF power simultaneously increased because the RF coupling with plasma improved due to the increase in the SOL density. In addition, the reflected power decreased. The OII line intensity and MoI line intensity also increased during the latter phase, but the $\mathrm{H}_{\alpha}$ line intensity remained constant. This suggests that the number of sput-
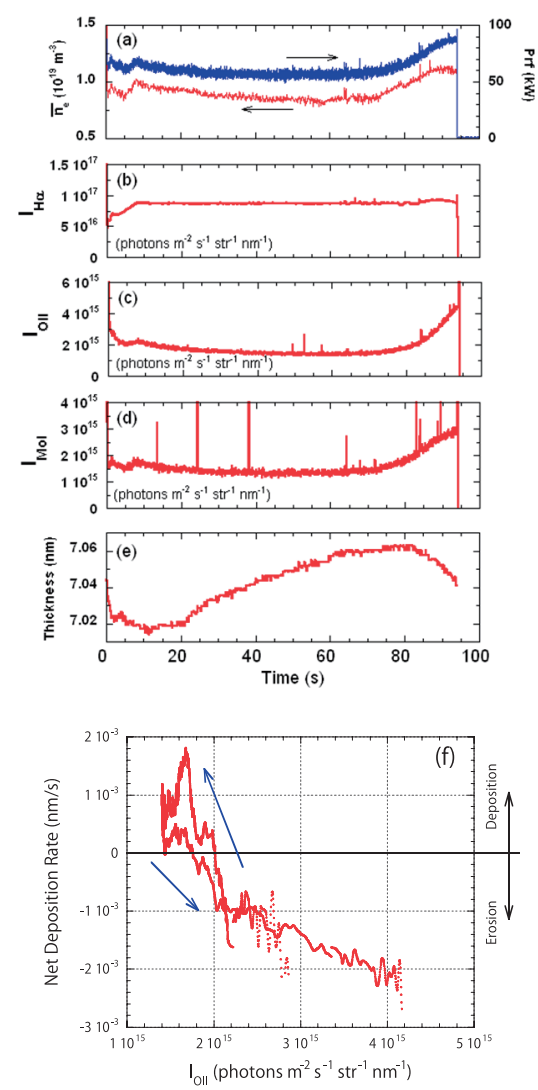

Fig. 7 Time evolution of (a) the line average electron density, (b) $\mathrm{H}_{\alpha}$ line intensity, (c) OII line intensity, (d) MoI line intensity, (e) thickness of deposited layer, and (f) deposition rate as a function of OII line intensity.

tered molybdenum atoms was increased by oxygen sputtering. It was found that the thickness of the deposited layer decreased in earlier and latter phases of the discharge (i.e., erosion phase), but increased in the middle phase of the discharge (i.e., deposition phase), as shown in Fig. 7 (e). Figure 7 (f) shows the deposition rate as a function of OII line intensity, which is considered to be related to oxygen flux. It seems that oxygen impurities affected the erosion and deposition properties, and a critical value exists for the oxygen flux during the transition from deposition to erosion, and vice versa. The deposition on the window may be expected to increase due to enhancement of sputtering on the divertor plate but simultaneously sputtering on the window also takes place. In addition, increase in SOL density prevents the transport of the molybdenum sputtered on the divertor plate. In a low density discharge (i.e., low oxygen amount), the deposition rate seems to depend on the ratio of molybdenum flux $\Gamma_{\mathrm{Mo}}$ to hydrogen flux $\Gamma_{\mathrm{H}}$, i.e. $\Gamma_{\mathrm{Mo}} / \Gamma_{\mathrm{H}}[17]$.

\subsection{Material analysis}

Material probe experiments were carried out in low density $\left(\sim 1 \times 10^{18} \mathrm{~m}^{-3}\right)$ and high density $\left(\sim 1 \times 10^{19} \mathrm{~m}^{-3}\right)$ long duration discharges [12-14]. The probe head with various types of specimens was inserted in SOL and ex- 
Table 1 Summary of the results of the material probe experiments and comparison between wall pumping rate, $\Gamma_{\text {wall }}^{\mathrm{MP}}$, estimated by ERD, and global wall pumping rate, $\Gamma_{\mathrm{wall}}^{\mathrm{GPB}}$, estimated by global particle balance analysis.

\begin{tabular}{|c|c|c|c|c|c|}
\hline & \multicolumn{2}{|c|}{ Low density $\left(\sim 1 \times 10^{18} \mathrm{~m}^{-3}\right)$} & \multicolumn{2}{|c|}{ High density $\left(\sim 1 \times 10^{19} \mathrm{~m}^{-3}\right)$} & \multirow[t]{2}{*}{ note } \\
\hline & E-side & P-side & E-side & P-side & \\
\hline Structure & bcc & fcc & bcc & bcc & \\
\hline Grain size & $10-20 \mathrm{~nm}$ & $1-2 \mathrm{~nm}$ & $10-20 \mathrm{~nm}$ & $10-20 \mathrm{~nm}$ & \\
\hline $\begin{array}{l}\text { Mo depo. } \\
\text { Rate }\end{array}$ & $\begin{array}{c}3.6 \times 10^{17} \\
\text { Mo } \mathrm{m}^{-2} \mathrm{~s}^{-1}\end{array}$ & $\begin{array}{c}6.4 \times 10^{16} \\
\text { Mo } \mathrm{m}^{-2} \mathrm{~s}^{-1}\end{array}$ & $\begin{array}{c}1.7 \times 10^{18} \\
\text { Mo } \mathrm{m}^{-2} \mathrm{~s}^{-1}\end{array}$ & $\begin{array}{c}2.3 \times 10^{18} \\
\text { Mo } \mathrm{m}^{-2} \mathrm{~s}^{-1}\end{array}$ & $\begin{array}{c}x=8 \mathrm{~mm} \\
\text { @E-side }\end{array}$ \\
\hline $\mathrm{H} / \mathrm{Mo}$ & 0.04 & 0.1 & 0.17 & 0.15 & \\
\hline$\Gamma_{\text {wall }}^{\mathrm{MP}}$ & $\begin{array}{l}1.3 \times 10^{16} \\
H \mathrm{~m}^{-2} \mathrm{~s}^{-1}\end{array}$ & $\begin{array}{l}6.4 \times 10^{15} \\
\mathrm{H} \mathrm{m}^{-2} \mathrm{~s}^{-1}\end{array}$ & 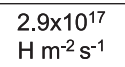 & $\begin{array}{l}3.5 \times 10^{17} \\
\mathrm{H} \mathrm{m}^{-2} \mathrm{~s}^{-1}\end{array}$ & \\
\hline \multirow{2}{*}{$\Gamma_{\text {wall }}^{\text {GPB }}$} & \multicolumn{2}{|c|}{$2 \times 10^{16} \mathrm{H} / \mathrm{m}^{2} \mathrm{~s}\left(\mathrm{HTW}^{*}\right)$} & \multirow{2}{*}{\multicolumn{2}{|c|}{$4.0 \times 10^{17} \mathrm{H} / \mathrm{m}^{2} \mathrm{~s}$}} & \multirow{2}{*}{$\begin{array}{l}\text { Whole wall } \\
\text { surface Is used. } \\
\left(\mathrm{S}=5 \mathrm{~m}^{2}\right)\end{array}$} \\
\hline & \multicolumn{2}{|c|}{$8.6 \times 10^{16} \mathrm{H} / \mathrm{m}^{2} \mathrm{~s}\left(\mathrm{LTW}^{*}\right)$} & & & \\
\hline
\end{tabular}

posed to long duration plasma. Then the following parameters were analyzed. The chemical composition and thickness of a deposited layer were estimated using Rutherford backscattering spectrometry (RBS), whereas the hydrogen concentration retained in the deposited layer was estimated using elastic recoil detection (ERD). The microstructure of the deposits was observed using transmission electron microscopy (TEM).

Table 1 summarizes the results [12-14]. The major element in the deposits was molybdenum. The grain size of the deposits was $1-2 \mathrm{~nm}$ or $10-20 \mathrm{~nm}$, but it depended on the setting position of the specimen as well as the plasma condition. It is found that $\mathrm{H} / \mathrm{Mo}$ of the co-deposits was rather higher than that of Mo bulk. The wall pumping rate, $\Gamma_{\text {wall }}^{\mathrm{MP}}$, which was estimated from the total amount of hydrogen retention in the co-deposits on the specimen and the discharge duration, seems to be consistent with the wall pumping rate estimated from the global particle balance $\Gamma_{\text {wall }}^{\mathrm{GPB}}$ using equation (1). $\Gamma_{\text {wall }}^{\mathrm{MP}}$ is the so-called static retention because it is estimated after the plasma discharge. On the other hand, $\Gamma_{\text {wall }}^{\mathrm{GPB}}$ represents the dynamic retention. Again, co-deposition of hydrogen with molybdenum is recognized as a possible candidate for the continuous wall pumping in long duration discharges.

The capability of the deposits to absorb hydrogen is correlated to the structure of the deposits [13]. Figure 8 (a) shows the thermal desorption spectra from the following three types of specimens: (i) Mo bulk, (ii) Mo deposits with fine grains shown in Fig. 8 (b), and (iii) Mo deposits with large grains shown in Fig. 8 (c). $D_{3}^{+}$with the energy of $6 \mathrm{keV}$ was implanted into each specimen at room temperature and the fluence was $1 \times 10^{21} \mathrm{D} / \mathrm{m}^{2}$. Figure $8(\mathrm{~d})$ shows the deuterium retention of each specimen, which was estimated from the thermal desorption spectra. As the grain size became smaller, the retention capability became higher. In deposits with fine grains, numerous effective trapping sites for hydrogen atoms, including grain boundaries or lattice defects, should be present. It should be noted that deposits with fine grains of Fig. 8 (b) were made under a vacuum condition with a little oxygen. As

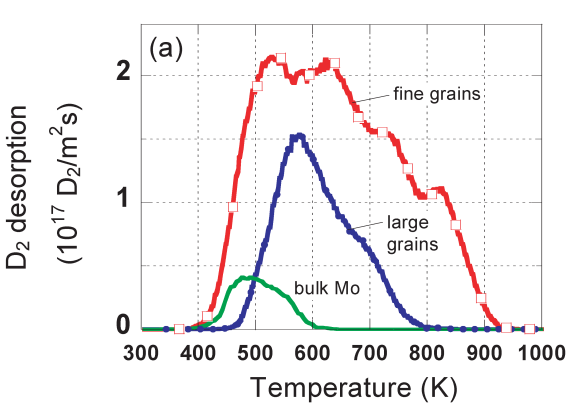

(b)
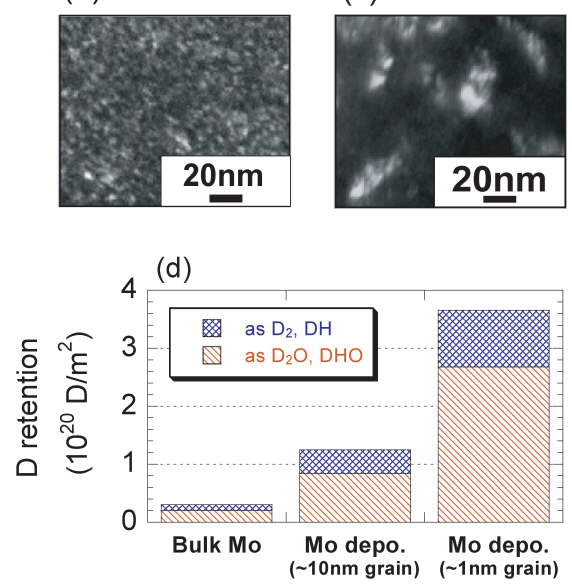

Fig. 8 Thermal desorption spectra from the molybdenum bulk and molybdenum deposits with fine grains and large grains. Dark field images of the microstructure of (b) fine grains and (c) large grains. (d) Deuterium retention of the three types of specimens.

reported in reference [4], the microstructure and grain size of the deposits are strongly affected by oxygen impurities. The oxygen atoms in the deposits seem to suppress free migration as well as crystallization of the deposited molybdenum atoms.

\section{Conclusion}

In TRIAM-1M, PWI extensive experiments were conducted and analyzed with an emphasis on the behaviors of hydrogen, molybdenum, and oxygen atoms from macroscopic and microscopic viewpoints. It is found that although the plasma density changes one order of magnitude, the change in the hydrogen recycling structure (i.e. toroidal profile of $\mathrm{H}_{\alpha}$ intensity) does not change so much. The characteristic decay length of the hydrogen recycling flux in the toroidal direction depends on $\bar{n}_{\mathrm{e}}^{-0.2}$. The density dependence of the decay length should be determined by neutral transport through SOL and the periphery region of the plasma during hydrogen recycling. The global wall pumping rate was estimated using global particle balance analysis. The wall temperature plays an important role in hydrogen re-emission from the wall. In the case of a high temperature wall, global wall saturation is observed during discharge, indicating that the hydrogen re-emission rate becomes higher than the hydrogen absorption rate dur- 
ing discharge. However, in the case of a low temperature wall, wall saturation is not observed until an ultra-long discharge with a duration of $5 \mathrm{~h} 16 \mathrm{~min}$. At this time, continuous growth of the deposited layer is observed from real time, in situ measurements of erosion and deposition. The microscopic approach using a material probe indicated that the major element in the deposits is molybdenum with a grain size of 1-2 nm or 10-20 nm. The hydrogen absorption rate of the deposits is consistent with the wall pumping rate estimated from particle balance analysis. Continuous wall pumping during a long duration discharge is attributed to the co-deposition of hydrogen with molybdenum. Oxygen impurities should affect the erosion and deposition properties on the wall as well as the hydrogen retention property of the deposited layer. The co-deposition of hydrogen with molybdenum and the oxygen impurity flux play important roles in the hydrogen recycling.

\section{Acknowledgements}

This work has been partially performed under the framework of joint-use research in RIAM Kyushu University and the bi-directional collaboration organized by NIFS. This work is partially supported by a Grant-in-Aid for Science Research from the Ministry of Education, Culture, Sports, Science and Technology.
[1] M. Shimada et al., J. Nucl. Mater. 337-339, 808 (2005).

[2] V. Philipps, Physica Scripta T123, 24 (2006).

[3] E. Tsitrone, J. Nucl. Mater. 363-365, 12 (2007).

[4] T. Hirai et al., J. Nucl. Mater. 283-287, 1177 (2000).

[5] T. Hirai et al., J. Nucl. Mater. 290-293, 94 (2001).

[6] M. Sakamoto et al., Nucl. Fusion 42, 165 (2002).

[7] M. Sakamoto et al., J. Nucl. Mater. 313-316, 519 (2003).

[8] Y. Hirooka et al., J. Nucl. Mater. 313-316, 588 (2003).

[9] M. Sakamoto et al., Nucl. Fusion 44, 693 (2004).

[10] H. Zushi et al., Nucl. Fusion 45, S142 (2005).

[11] K. Hanada et al., Fusion Eng. Des. 81, 2257 (2006).

[12] M. Miyamoto et al., J. Nucl. Mater. 313-316, 82 (2003).

[13] M. Miyamoto et al., J. Nucl. Mater. 337-339, 436 (2005).

[14] M. Tokitani et al, J. Nucl. Mater. 367-370, 1487 (2007).

[15] M. Ogawa et al., J. Nucl. Mater. 363-365, 1364 (2007).

[16] R. Bhattacharyay et al., Nucl. Fusion 47, 864 (2007).

[17] M. Sakamoto et al., J. Nucl. Mater. 363-365, 233 (2007).

[18] E. Jotaki and S. Itoh, Fusion Eng. Des. 36, 447 (1997).

[19] K. Hanada et al., Fusion Eng. Des. 54, 79 (2001).

[20] D. Heifetz, D. Post, M. Petravic et al., J. Comput. Phys. 46, 309 (1982).

[21] E. Tsitrone et al., Plasma Phys. Control. Fusion 44, 701 (2002).

[22] T. Loarer et al., Nucl. Fusion 47, 1112 (2007).

[23] T. Nakano et al., J. Nucl. Mater. 363-365, 1315 (2007).

[24] T. Nakano et al., Nucl. Fusion 48, 085002 (2008).

[25] F. Weschenfelder, P. Wienhold and J. Winter, J. Nucl. Mater. 196-198, 1101 (1992). 